\title{
Atividade nictimeral e tempo de digestão de Aegla longirostri (Crustacea, Decapoda, Anomura)
}

\author{
Carolina C. Sokolowicz ${ }^{1}$, Luciane Ayres-Peres ${ }^{2} \&$ Sandro Santos $^{2}$
}

1. Programa de Pós-Graduação em Biologia Animal, Departamento de Zoologia, Universidade Federal do Rio Grande do Sul, Avenida Bento Gonçalves, 9500, prédio 43435, 91501-970 Porto Alegre, RS, Brasil. (carolinasokolowicz@ hotmail.com)

2. Departamento de Biologia, Centro de Ciências Naturais e Exatas, Universidade Federal de Santa Maria, 97105-900 Santa Maria, RS, Brasil. (lucianeayres@ hotmail.com, ssantos@smail.ufsm.br)

\begin{abstract}
Diel activity and digestion time of Aegla longirostri (Crustacea, Decapoda, Anomura). The aim of this work was to characterize the diel activity rhythm and time of digestion in Aegla longirostri Bond-Buckup \& Buckup, 1994. The individuals were collected in Santa Maria, RS, Brazil. In laboratory, the animals were kept in individuals glass aquariums (5L). To test locomotor and feeding activity of the aeglids, a group remained under constant luminosity for 12 hours, while another group was mantained in the dark, this condition being reversed at each 12 hours. The observations were taken at every 6 hours. For the determination of digestion's time the animals were fed, and one individual was sacrificed at each 30 minutes. Aegla longirostri showed lower activity in periods of light time. This condition was recorded for both groups of animals. This species takes approximately 5 hours to conclude its extracellular digestion. The results suggest that $A$. longirostri has nocturnal habits and probably feeds whenever resources are available.
\end{abstract}

KEYWORDS. Activity pattern, extracellular digestion, Aeglidae.

RESUMO. O objetivo do presente trabalho foi caracterizar o ritmo de atividade e tempo de digestão em Aegla longirostri Bond-Buckup \& Buckup, 1994. Os animais foram coletados em Santa Maria, RS, Brasil. Em laboratório, os animais foram transferidos para aquários individuais (5L). Para testar a atividade locomotora e alimentar dos aeglídeos um grupo de animais permaneceu sob luminosidade constante durante 12 horas, enquanto outro grupo permanecia no escuro, essa condição sendo invertida a cada 12 horas. As observações foram realizadas a cada 6 horas. Para determinar o tempo de digestão os animais foram alimentados e a cada 30 minutos um indivíduo era sacrificado. Aegla longirostri mostrou menor atividade em períodos de luminosidade, o que aconteceu nos dois grupos de animais. Essa espécie leva aproximadamente 5 horas para concluir a digestão extracelular. Os resultados sugerem que A. longirostri possui hábitos noturnos e provavelmente se alimenta sempre que há recursos disponíveis.

PALAVRAS-CHAVE. Padrão de atividade, digestão extracelular, Aeglidae.

A exposição à luz pode afetar diversos processos fisiológicos nos crustáceos, tais como a locomoção, alimentação, acasalamento, mudança de cor e metabolismo (MEYER-Rochow, 1994). O estudo sobre o comportamento dos crustáceos em relação aos períodos de luz e escuridão, assim como a estimativa do tempo de digestão, é essencial para o conhecimento da biologia do grupo. Através de tais estudos, pode-se demonstrar o período do dia em que o animal apresenta maior atividade, inclusive alimentar, permitindo inferir sobre período de forrageamento e maior suscetibilidade a predadores.

Investigações que levam em consideração o ritmo de atividade em crustáceos têm sido realizadas freqüentemente com caranguejos e camarões, destacando-se os estudos de PAlmer (1971), que analisou o padrão de atividade de três espécies de braquiúros e um anomuro semi-terrestre. REBACH (1974) estudou a influência da temperatura na atividade de escavação do ermitão Pagurus longicarpus Say, 1817. BARNES (1997) observou a atividade de Coenobita rugosus H. Milne Edwards, 1837 e C. cavipes Stimpson, $1858 \mathrm{em}$ função da luminosidade. REIGADA (2002) investigou a atividade nictimeral durante o período de claro e escuro em Callinectes ornatus Ordway, 1863 e C. danae Smith, 1869; WILLINER \& ColLins (2002) estudaram a variação espaçotemporal da atividade de Macrobrachium jelskii (Miers, 1877). Mais recentemente, Pontes \& ARruda (2005) analisaram o comportamento de Litopenaeus vannamei (Boone, 1931) durante as fases clara e escura.

Os aeglídeos são crustáceos peculiares por serem os únicos anomuros de águas continentais. Este é um grupo endêmico do sul da América do Sul, restrito à região temperada e subtropical, ocorrendo em arroios, rios de correnteza e lagos, desde o município de Franca, SP, Brasil, até a Ilha Madre de Díos, no Chile (BOND - BuCKUP \& BUCKUP, 1994). Estes anomuros têm papel fundamental, por serem elos nas cadeias alimentares dos ambientes limnícos, integrando a dieta de peixes, rãs e aves (ARENAS, 1976) e predando larvas de alguns insetos (MAGNI \& Py-DANIEL, 1989).

Aegla longirostri Bond-Buckup \& Buckup, 1994 é uma espécie de distribuição restrita ao nordeste, centro e leste do Estado do Rio Grande do Sul, Brasil (Bond-BuCKuP $\&$ BuCKuP, 1999). O presente estudo teve como objetivo analisar, em laboratório, a atividade nictimeral de $A$. longirostri, avaliando a influência da luminosidade em suas atividades locomotoras e alimentares, e estimar o tempo de digestão extracelular da espécie.

\section{MATERIAL E MÉTODOS}

Atividade nictimeral. Os aeglídeos foram coletados manualmente e com auxílio de puçá, em julho de 2002, no município de Santa Maria, RS, Brasil, em arroio tributário do Rio Vacacaí-Mirim (2940'13”S, 5345'44”'W). No Laboratório de Carcinologia, Departamento de Biologia (CCNE-UFSM), os crustáceos permaneceram individualizados em aquários $(30 \times 15 \times 20 \mathrm{~cm})$ com rochas retiradas do local de coleta e aeração constante, passando por um período de aclimatação de 5 dias. 
Para testar a atividade locomotora e alimentar de $A$. longirostri, os animais, todos em intermuda, foram divididos em dois grupos de seis indivíduos. Um grupo permaneceu com luminosidade constante por 12 horas, enquanto o outro ficou sem iluminação, sempre um grupo seguindo o fotoperíodo normal $(\mathrm{FN})$ do dia e outro permanecia em fotoperíodo inverso (FI), sendo essa condição invertida a cada de 12 horas. Para possibilitar a observação do comportamento dos animais no período de escuridão, foram utilizadas lâmpadas incandescentes vermelhas, amplamente utilizadas em trabalhos de observação noturna em crustáceos (Turra \& Denadai, 2003). Durante a realização dos experimentos, a cada observação, foram feitas medidas da temperatura da água nos aquários.

As observações foram conduzidas em intervalos de seis horas (com início às $2 \mathrm{~h}$ ) durante sete dias. Em cada observação o comportamento dos animais foi acompanhado por 30 minutos; registrando-se o tempo em que se deslocavam, o tempo em que se mantinham abrigados nas rochas ou embaixo do aerador, e o período em que ficavam desabrigados.

Os dois grupos foram alimentados diariamente, com filé de peixe, às $14 \mathrm{~h}$, durante 25 minutos. Após esse tempo o restante do alimento foi retirado. Foi observado quanto tempo os animais permaneciam em atividade alimentar.

Para testar possíveis diferenças significativas do deslocamento e alimentação dos animais nos períodos de claro/escuro, nos grupos FN e FI separadamente, foi aplicado o teste $t$ de "Student" (ZAR, 1996).

Tempo de digestão. Para o estudo do tempo de digestão, os experimentos foram realizados em replicata. Foram realizadas duas coletas manuais de A. longirostri, nos meses de julho/2001 (experimento I) e agosto/2001 (experimento II). Os indivíduos coletados, todos em intermuda, foram acondicionados em uma caixa térmica contendo água e rochas retiradas do local e transportados para o laboratório. Foram realizadas medidas da temperatura da água nos aquários para mantê-la o mais próximo possível da temperatura do ambiente natural.

Nesse estudo, todos os animais utilizados estavam no período de intermuda, isto é, o intervalo de tempo entre uma muda e outra. Durante a fase de pós muda, antes do novo exoesqueleto tornar-se rígido, o animal torna-se vulnerável a danos como predação e estresse osmótico. Nesse período, podem permanecer reclusos, escondendose em locais protegidos, sem se alimentar (BRUSCA \& BRUSCA, 2003), bem como na fase de pré muda, em que os crustáceos deixam de se alimentar e diminuem sua atividade. Pelo fato de os períodos que antecedem e se seguem a muda serem fases de grande estresse para os crustáceos, esses animais não foram utilizados nesse estudo, pois poderiam comprometer os resultados obtidos.

No laboratório, os aeglídeos foram individualizados em aquários $(30 \times 15 \times 20 \mathrm{~cm})$ contendo rochas e aeração constante. Os animais permaneceram nos aquários em aclimatação durante 48 horas sem receber alimento, para que evacuassem totalmente o conteúdo dos estômagos.
Em cada experimento foram utilizados 11 animais. Para cada um foi oferecido cerca de 0,4 grama de filé de peixe. $\mathrm{O}$ alimento permaneceu nos aquários por um período de uma hora, em seguida o restante foi retirado. Na seqüência, a cada 30 minutos, um indivíduo foi sacrificado por meio de congelamento em freezer.

O congelamento dos animais é uma técnica amplamente utilizada em estudos que levam em conta os itens alimentares na dieta de crustáceos (BROGIM \& LANA, 1997; GuAn \& Wiles, 1998; Mantelatto \& Christofoletti, 2001; Albertoni et al., 2003); e, segundo Mantelatto \& Christofoletti (2001), em seu estudo com Callinectes ornatus, o congelamento dos animais imediatamente após a captura é utilizado para retardar a digestão e cessar a maceração no moinho gástrico.

Após o descongelamento cada animal foi dissecado; o estômago foi removido e observado sob estereomicroscópio. Para que houvesse padronização da observação dos estômagos, o Grau de Repleção Estomacal (G.R.) foi registrado, comparando-se o volume de alimento presente com o volume total do estômago. Os estômagos foram classificados em quatro categorias (modificado de WILLIAMS, 1981): zero = estômago totalmente vazio; 0,25 = até $25 \%$ do estômago ocupado pelo alimento; $0,5=$ entre 26 e $50 \%$ e $1=$ entre 51 e $100 \%$.

\section{RESULTADOS}

Atividade nictimeral. Foram feitas 14 horas de observação para cada fotoperíodo (FN, FI). A temperatura da água nos aquários variou de $11,5^{\circ} \mathrm{C}$ a $16^{\circ} \mathrm{C}$, como observada em ambiente natural nesse período do ano (inverno), na região estudada.

A maior atividade locomotora $(20,16 \%)$ no grupo $\mathrm{FN}$ foi registrada às $2 \mathrm{~h}$ (escuro) e no FI às $8 \mathrm{~h}(18,57 \%)$, também no escuro. Os menores valores de atividade locomotora foram observados no fotoperíodo normal $(3,65 \%)$ às $14 \mathrm{~h}$ (claro) e no inverso $(0,08 \%)$ às $2 \mathrm{~h}$ (claro) (Fig. 1$)$.

Houve diferença significativa $(\mathrm{p}<0,01)$ entre os valores de deslocamento nos períodos de claro e escuro, para os dois grupos. Aegla longirostri apresentou maior atividade locomotora no período de menor luminosidade, padrão corroborado no grupo mantido com fotoperíodo inverso.

Observou-se, em ambos fotoperíodos, que durante os períodos em que os animais não estavam expostos à luz, permaneciam mais tempo longe das rochas (deslocando-se ou parados) do que abrigados sob ou próximos às mesmas (Tab. I).

Não houve diferença significativa entre a atividade alimentar para os dois grupos ( $\mathrm{p}>0,05)$. Os animais do grupo FI passaram $74,8 \%$ do tempo se alimentando, e os do FN, $76,2 \%$ do tempo em que o alimento estava disponível.

Tempo de digestão. Foram examinados 10 fêmeas e 12 machos. O tempo que A. longirostri levou para digerir extracelularmente o alimento, ou seja, o tempo necessário para que os estômagos ficassem totalmente vazios (G.R.= zero), em ambos os experimentos, foi de até 5 horas (Figs. 2, 3).

Tabela I. Porcentagem (\%) de Deslocamento (D), tempo Fora da Rocha (FR) e Embaixo ou próximo a Rocha (ER) de Aegla longirostri Bond-Buckup \& Buckup, 1994 nos quatro horários de observação, nos fotoperíodos Normal e Inverso.

\begin{tabular}{|c|c|c|c|c|c|c|c|c|}
\hline \multirow[t]{2}{*}{ Horário } & \multicolumn{2}{|c|}{ 02:00 } & \multicolumn{2}{|c|}{ 08:00 } & \multicolumn{2}{|c|}{$14: 00$} & \multicolumn{2}{|c|}{ 20:00 } \\
\hline & Normal\% & Inverso $\%$ & Normal\% & Inverso $\%$ & Normal\% & Inverso $\%$ & Normal\% & Inverso $\%$ \\
\hline$\overline{\mathrm{D}}$ & 20,16 & 0,08 & 6,27 & 18,57 & 3,65 & 6,27 & 17,07 & 0,32 \\
\hline FR & 36,42 & & 13,97 & 13,25 & 9,92 & 26,67 & 25,79 & 0,16 \\
\hline ER & 43,42 & 99,92 & 79,76 & 68,18 & 86,43 & 67,06 & 57,14 & 99,52 \\
\hline
\end{tabular}




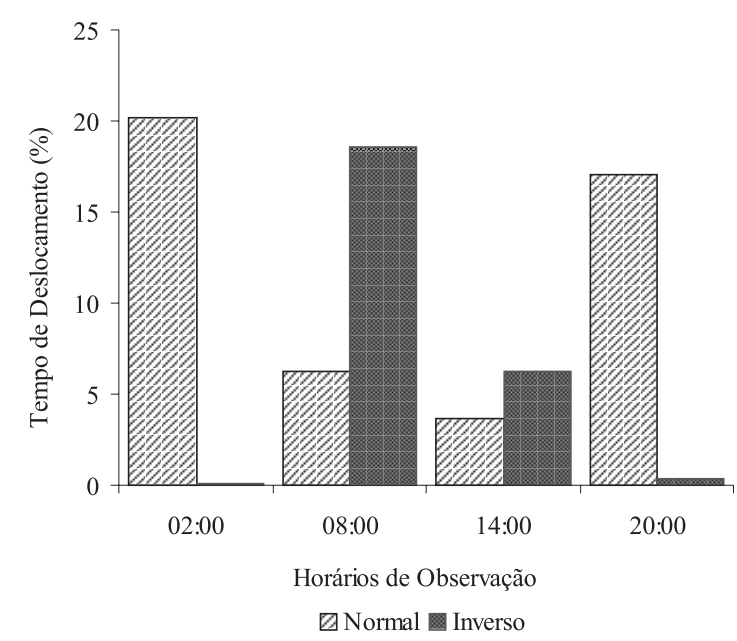

Fig. 1. Porcentagem de deslocamento de Aegla longirostri BondBuckup \& Buckup, 1994 nos quatro horários de observação no fotoperíodo normal e inverso.
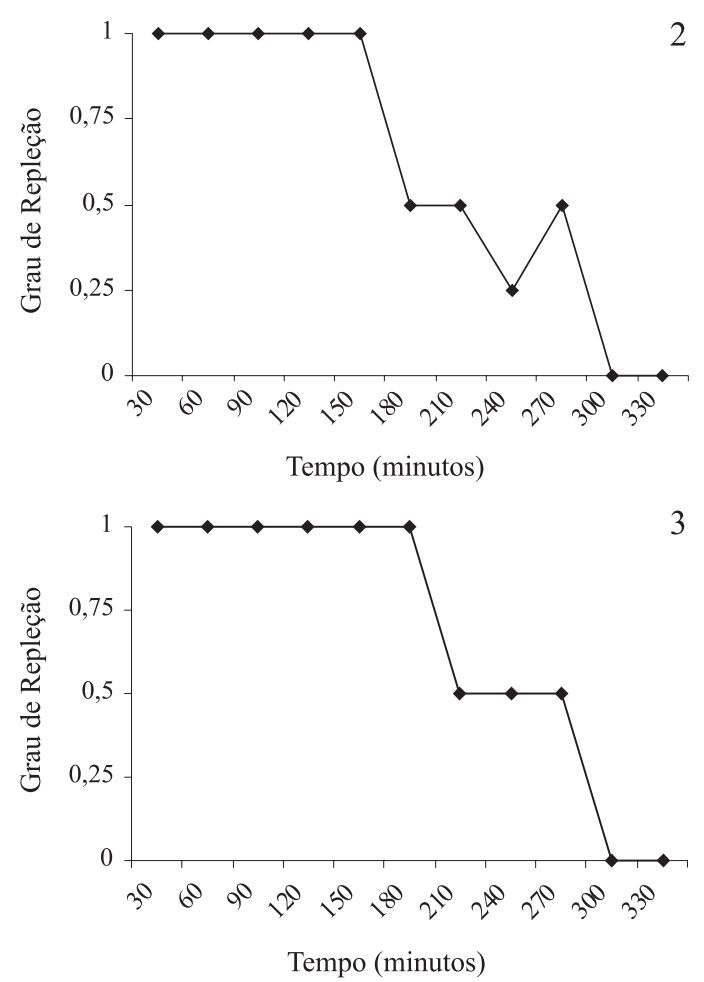

Figs. 2, 3. Tempo de digestão em função do grau de repleção de cada estômago de Aegla longirostri Bond-Buckup \& Buckup, 1994, em condições de laboratório: 2, experimento I; 3, experimento II.

\section{DISCUSSÃO}

A maior atividade locomotora observada no período de menor luminosidade indica que $A$. longirostri apresenta hábitos preferencialmente noturnos. Porém, a atividade alimentar pode ser independente da fotofase (claro/escuro), ou seja, sempre que houve alimento disponível os animais fizeram uso do mesmo.

ZIMMER-FAusT et al. (1996), trabalhando com o siri azul, Callinectes sapidus Rathbun, 1896, verificaram que os animais apresentam um ritmo de atividade endógeno, no qual o comportamento locomotor ocorre principalmente à noite, porém, esse modelo pode mudar na presença de estímulo químico. REIGADA (2002) observou que para os siris Callinectes ornatus e $C$. danae, a atividade do animal na presença do alimento também é alterada, independente do fotoperíodo. PONTES \& ARRUDA (2005) verificaram que, para o camarão Litopenaeus vannamei, a alimentação foi mais freqüente nos períodos de luminosidade. No entanto, também observaram que a exploração do substrato foi intensificada após a disponibilidade de alimento em ambos fotoperíodos, indicando uma relação com a busca de alimento. $\mathrm{O}$ fato de que para $A$. longirostri os animais tornaram-se ativos tanto na fase clara como na escura na presença de alimento pode indicar um comportamento semelhante, sugerindo que a presença de alimento desencadeia a atividade.

BuEno \& Bond-Buckup (2004), em estudo sobre ecologia alimentar de Aegla platensis Schmitt, 1942 e A. ligulata Bond-Buckup \& Buckup, 1994 coletaram os animais em diversos horários e analisaram o conteúdo estomacal, concluindo que, no horário das $24 \mathrm{~h}$, A. platensis apresentou maior grau de repleção estomacal, indicando uma tendência maior da atividade alimentar à noite. Aegla ligulata mostrou maior atividade alimentar ao entardecer, com altos valores de repleção estomacal às $18 \mathrm{~h}$.

A atividade noturna também é conhecida para outros crustáceos. PALMER (1971), nas observações em campo e em condições controladas no laboratório, observou que a atividade locomotora dos caranguejos Geocarcinus lateralis (Freminville, 1835), Cardisoma guanhumi (Latreille, 1825), Ocypode quadrata (Fabricius, 1787) e o anomuro semi-terrestre Coenobita clypeatus (Herbst, 1794) ocorrem no escuro. $\mathrm{O}$ autor também constatou que a atividade locomotora foi independente da temperatura.

Em observações preliminares, em que a temperatura nos aquários variou de $26 \mathrm{a} 30^{\circ} \mathrm{C}$, foram obtidos os mesmos resultados que nesse trabalho, corroborando os resultados de PALmer (1971).

Para Callinectes ornatus e $C$. danae, foi observado que indivíduos de ambas as espécies apresentaram maior atividade, ou seja, ficaram mais tempo sobre o sedimento (desenterrados) durante os períodos de baixa intensidade luminosa (REIGADA, 2002). O mesmo também ocorre em Litopenaeus vannamei (PONTES \& ARRUDA, 2005).

É conhecido que os aeglídeos servem de fonte alimentar para aves, rãs, peixes e jacarés (ARENAS, 1976), todos animais com orientação visual e hábitos preferencialmente diurnos. Considerando-se estes fatos, pode-se inferir que o hábito noturno de A. longirostri representa uma das estratégias de adaptação destes anomuros para evitar a predação.

Entre os trabalhos em que se analisou o tempo de digestão de crustáceos, com base na digestão de tecidos animais, destacam-se os de HiLl (1976), que observou o tempo de digestão de Scylla serrata (Forskal, 1775) em no máximo 12 horas, PAUL (1981), que registrou o tempo de 6 horas para que Callinectes arcuatus Ordway, 1836 evacuasse completamente o conteúdo do seu estômago, e CHоу (1986), o qual, estudando Liocarcinus puber (Linnaeus, 1767) e L. holsatus (Fabricius, 1798), registrou um tempo de 25 horas para a digestão completa nessas espécies.

Além do item alimentar a ser digerido e da espécie em questão, outros fatores também podem influenciar o tempo de digestão de um crustáceo, como por exemplo a temperatura do ambiente. Espécies tropicais e subtropicais apresentam um metabolismo mais rápido do que espécies de clima 
temperado (CHоY, 1986). A temperatura da água nos aquários, durante os dois experimentos, ficou em $16 \pm 2^{\circ} \mathrm{C}$, semelhante à temperatura do ambiente natural, para esta época do ano no Rio Grande do Sul, Brasil, $13^{\circ} \mathrm{C}$ no inverno e $22^{\circ} \mathrm{C}$ no verão. Essas médias foram estimadas através de medidas mensais da temperatura durante 15 meses de amostragem (BUENO \& BOND-BuCKuP, 2000).

Considerando-se o hábito noturno dos animais e o tempo de digestão, é possível inferir que $A$. longirostri deixa o abrigo para se alimentar ao entardecer. Levando-se em conta que o animal não se alimente enquanto todo o alimento não for digerido (o que deverá ocorrer por volta de meia noite), provavelmente em seguida à digestão, ele é capaz de realizar outro processo de busca por alimento antes que o dia comece a clarear novamente, quando então ele busca abrigo sob as rochas (Fig. 4).

Corroborando a hipótese levantada podemos citar o trabalho de WolfF \& CERDA (1992), com Cancer polyodon Poeppig, 1836, um braquiúro chileno. Os autores concluíram que a maior atividade alimentar ocorre à noite, porém os caranguejos cessam a alimentação ao nascer do sol ou apenas a diminuem, e voltam a se alimentar quando os resíduos são eliminados, o que acontece por volta das 6 horas.

Os resultados obtidos entre associação de um estudo comportamental com o experimento de tempo de digestão podem traçar uma hipótese a respeito do ciclo diário de $A$. longirostri, permitindo inferir sobre aspectos como períodos de maior e menor atividade e forrageamento, essas informações podem ser importantes em futuros estudos a serem desenvolvidos não apenas com A. longirostri, mas com outros membros da família Aeglidae.

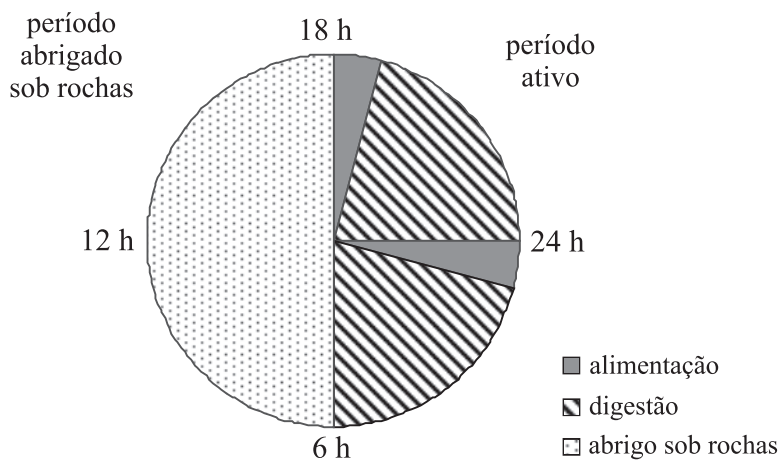

Fig. 4. Hipótese para o ciclo diário de atividades de Aegla longirostri Bond-Buckup \& Buckup, 1994

Agradecimentos. Aos Biólogos Maríndia Deprá, Sonia Cechin, Alberto S. Gonçalves e Cássio F. Puerari, pelo auxílio durante a análise preliminar desse trabalho. Aos Biólogos Marcia R. Spies e Tiago Gomes dos Santos, pelas sugestões e críticas ao trabalho.

\section{REFERÊNCIAS BIBLIOGRÁFICAS}

Albertoni, E. F.; Palma-Silva, C. \& Esteves, F. DE A. 2003. Overlap of dietary niche and electivity of three shrimp species (Crustacea, Decapoda) in a tropical coastal lagoon (Rio de Janeiro, Brazil). Revista Brasileira de Zoologia 20(1):135-140.

ARENAS, J. 1976. La cordillera de la costa como refugio de la fauna dulcícola preglacial. Archivos de Biologia y Medicina Experimentales 10:1-40.
BARNES, D. K. A. 1997. Ecology of tropical hermit crabs at Quirimba Island, Mozambique: distribution, abundance and activity. Marine Ecology Progress Series 154:133-142.

Bond-Buckup, G. \& Buckup, L. 1994. A família Aeglidae (Crustacea, Decapoda, Anomura). Arquivos de Zoologia 32(4):159-346. .1999. Família Aeglidae (caranguejos anomuros de água doce). In: Buckup, L. \& Bond-Buckup, G. eds. Os crustáceos do Rio Grande do Sul. Porto Alegre, UFRGS. p.362-382.

Brogim, R. A. \& LanA, P. C. 1997. Espectro alimentar de Aratus pisonii, Chasmagnathus granulata e Sesarma rectum (Decapoda, Grapsidae) em um manguezal da Baía de Paranaguá. Iheringia, Série Zoologia, 83:35-44.

Brusca, R. C. \& Brusca, G. J. 2003. Invertebrates. 2 ed. Sunderland Sinauer. $936 \mathrm{p}$

Bueno, A. A. P. \& Bond-Buckup, G. 2000. Dinâmica populacional de Aegla platensis Schmitt (Crustacea, Decapoda, Aeglidae). Revista Brasileira de Zoologia 17(1):43-49.

2004. Natural diet of Aegla platensis Schmitt and Aegla ligulata Bond-Buckup \& Buckup (Crustacea, Decapoda, Aeglidae) from Brazil. Acta Limnológica Brasileira 16(2):115-127.

CHoy, S. C. 1986. Natural diet and feeding habits of the crabs Liocarcinus puber and L. holsatus (Decapoda, Brachyura, Portunidae). Marine Ecology Progress Series 31:87-99.

Guan, R.-Z. \& Wiles, P. R. 1998. Feeding ecology of the signal crayfish Pacifastacus leniusculus in a British lowland river. Aquaculture 169(3):177-193.

HILL, B. J. 1976. Natural food, foregut clearance-rate and activity of the crab Scylla serrata. Marine Biology 34(2):109-116.

Magni, S. T. \& Py-Daniel, V. 1989. Aegla platensis Schmitt, 1942 (Decapoda, Anomura) um predador de imaturos de Simuliidae (Diptera Culicomorpha). Revista de Saúde Pública 23(3):258-259.

Mantelatto, F. L. M. \& Christofoletti, R. A. 2001. Natural feeding activity of the crab Callinectes ornatus (Portunidae) in Ubatuba Bay (São Paulo, Brazil): influence of season, sex, size and molte stage. Marine Biology 138(3):585-594.

Meyer-Rochow, V. B. 1994. Light-induced damage to photoreceptors of spiny lobsters and other crustaceans. Crustaceana 67:95-109.

Palmer, J. D. 1971. Comparative studies of circadian locomotory rhythms in four species of terrestrial crabs. American Midland Naturalist 85(1):97-107.

PAUL, R. K. G. 1981. Natural diet, feeding and predatory activity of the crabs Callinectes arcuatus and $C$. toxotes (Decapoda, Brachyura, Portunidae). Marine Ecology Progress Series 6:91-99.

Pontes, C. S. \& Arruda, M. F. 2005. Comportamento de Litopenaeus vannamei (Boone) (Crustacea, Decapoda, Penaeidae) em função da oferta de alimento artificial nas fases clara e escura do período de 24 horas. Revista Brasileira de Zoologia 22(3):648-652.

REBACH, S. 1974. Burying behavior in relation to substrate and temperature in the hermit crab, Pagurus longicarpus. Ecology 55(1):195-198.

Reigada, A. L. D. 2002. Diel activity rhythm in Callinectes ornatus Ordway, 1863 and Callinectes danae Smith, 1869 (Brachyura, Portunidae) under laboratory conditions. In: EscoBAR-BRIONES, E. \& Alavarez, F. orgs. Modern Approaches to the Study of Crustacea. Norwell, Kluwer Academic. p.915-920.

Turra, A. \& Denadai, M. R. 2003. Daily activity of four tropical intertidal hermit crabs from southeastern Brazil. Brazilian Journal of Biology 63(3):537-544.

Williams, M. J. 1981. Methods for analysis of natural diet in portunid crabs (Crustacea: Decapoda: Portunidae). Journal of Experimental Marine Biology and Ecolology 52:103-113.

Williner, V. \& Collins, P. 2002. Variación espacio-temporal de la actividad del camaron dulceacuicola Macrobrachium jelskii (Miers, 1877) (Crustacea, Decapoda, Caridea, Palaemonidae). Ecologia-Austral 12(1):3-10.

WolfF, M. \& Cerda, G. 1992. Feeding ecology of the crab Cancer polyodon in La Herradura Bay, Northern Chile. I. Feeding chronology, food intake, and gross growth and ecology efficiency. Marine Ecology Progress Series 89:213-219.

ZAR, J. H. 1996. Biostatistical Analysis. New Jersey, PrenticeHall. 907p.

Zimmer-Faust, R. K.; O’ Neill, B. \& Schar, D. W. 1996. The relationship between predador activity state and sensitivity to prey odor. Biological Bulletin 190(1):82-87. 